\title{
Conical Nanoantenna Arrays for Terahertz Light
}

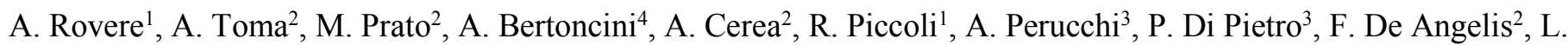 \\ Manna $^{2}$, R. Morandotti ${ }^{1}$, C. Liberale ${ }^{4}$, L. Razzari ${ }^{1}$ \\ ${ }^{1}$ INRS - Énergie, Matériaux et Télécommunications, 1650 Blvd Lionel Boulet, J3X 1S2 Varennes (Québec), Canada \\ ${ }^{2}$ Fondazione Istituto Italiano di Tecnologia, via Morego 30, 16163 Genova, Italy \\ ${ }^{3}$ INSTM UdR Trieste-ST and Sincrotrone Trieste, Area Science Park, Basovizza, 34012 Trieste, Italy \\ ${ }^{4}$ KAUST, BESE (Biological Environmental Science and Engineering) division, Thuwal 23955-6900, Saudi Arabia.
}

\begin{abstract}
We report here on the design, fabrication and characterization of out-of-plane arrays of conical nanoantennas resonating in the terahertz frequency range.
\end{abstract}

Keywords-Terahertz Nanoantennas; $3 D$ nanostructures; Enhanced Spectroscopy.

\section{INTRODUCTION}

In recent years, planar 2D nanoantennas (NAs) over transparent substrates have shown to be a viable solution for concentrating freespace radiation beyond the diffraction limit thus leading to a local field enhancement that can be used to intensify light-matter interaction [1]. Recently, these properties have been exploited in the terahertz (THz) frequency range $(0.1-10 \mathrm{THz})$, through the development of Nanoantenna Enhanced THz Spectroscopy (NETS) [2] - a technique that allows sensing a broad range of elementary excitations in tiny quantities of nanomaterials and molecules. Despite these unique capabilities, planar NAs still present significant limitations when employed in surface-enhanced spectroscopy, since they feature extremely broad resonances with poor enhancement properties and furthermore it is not straightforward to effectively localize an analyte in proximity of their electromagnetic "hot spots" (usually located at the NA's extremities). To circumvent these difficulties, more recently, the use of vertical 3D nanostructures has been proposed [3]. In an array configuration, the lateral scattering can lead to a strong constructive coupling between neighbouring 3D NAs, resulting in a more pronounced far-field response and a higher field enhancement. Moreover, vertical structures present more accessible "hot spots" and can also be used as resonant probes for tip-enhanced spectroscopies [4]. Here we present a preliminary investigation on the frequency response of $3 \mathrm{D}$ gold nanocone (NC) arrays resonating at $\mathrm{THz}$ frequencies. First, we performed numerical simulations to optimize the electromagnetic response of the nanostructures. Then, we fabricated the samples and characterised them in the $\mathrm{THz}$ region. Experimental results are in fairly good agreement with the numerical simulations and further investigations are planned.

\section{NUMERICAL AND EXPERIMENTAL RESULTS}

By means of electromagnetic simulations (CST Microwave Studio), we considered various gold NCs arrays positioned over a gold surface and covering an area of $100 \times 100 \mu \mathrm{m}^{2}$. The height and base radius of the NCs were fixed at $9 \mu \mathrm{m}$ and $450 \mathrm{~nm}$, respectively, in order to set the main resonance of an isolated $\mathrm{NC}$ at around $8 \mathrm{THz}$ (our target frequency for a proof of principle demonstration of $\mathrm{NC}$ enhanced spectroscopy, see below). Since our NCs are placed over a conductive surface, they exhibit a monopole-like response, which results in a charge density at the NC apex that is doubled with respect to that of a dipole, thus further increasing the local electric field [3].
Arrays featuring different pitches were simulated, in order to optimize the NCs' mutual interaction and their field enhancement at the target frequency. Based on these numerical results, NC arrays were fabricated using advanced $3 \mathrm{D}$ printing based on two-photon

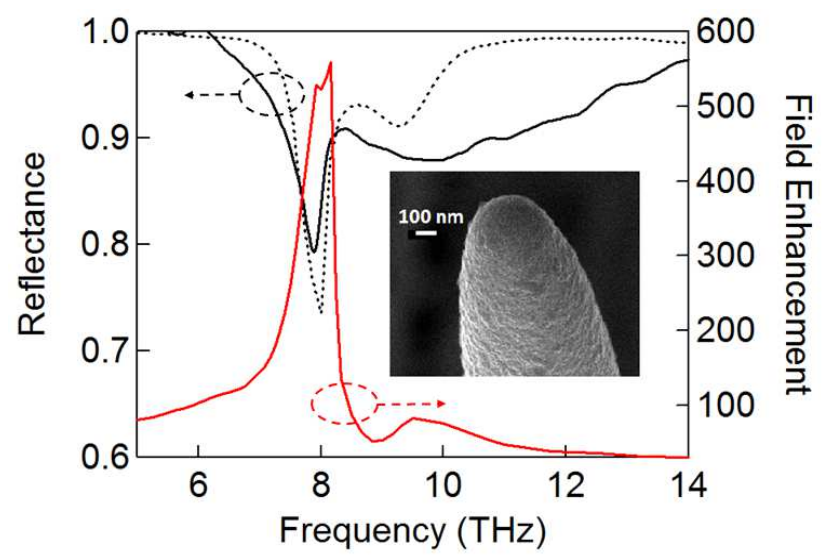

Fig. 1. Characterization of an array of $100 \times 100 \mu \mathrm{m}^{2}$ of $3 \mathrm{D}$ gold cones, with height and pitch of $9 \mu \mathrm{m}$ and $25 \mu \mathrm{m}$, respectively. Measured (solid black) and simulated (dashed) reflectance of the sample. The red curve shows the field enhancement calculated in proximity of the tip of a cone (SEM image in the inset).

lithography (Nanoscribe), a technology that is able to pattern complex $3 \mathrm{D}$ polymeric nanostructures. The inset in Fig. 1 shows the SEM image of the tip of a NC, after a 50-nm-thick gold layer was finally evaporated over the array. A radius of curvature of only $100 \mathrm{~nm}$ after evaporation was achieved, which is expected to lead to field enhancement values up to several hundreds (see, for example, the simulated red solid curve in Fig. 1). Finally, the samples were characterized using broadband synchrotron radiation (Elettra facility Trieste, Italy). Reflection spectra were collected between 5 and 14 $\mathrm{THz}$, and $\mathrm{THz}$ radiation was focused on the sample area through a Cassegrain microscope. Fig. 1 (black solid line) shows the reflectance of a NC array with pitch of $25 \mu \mathrm{m}$. We observe a fair agreement between this result and what predicted by numerical simulations (dashed black curve). Further experiments are planned to explore the potential of these substrates for the characterization of colloidal cadmium sulfide nanocrystals, which possess a phonon resonance centered at $7.8 \mathrm{THz}$.

\section{REFERENCES}

[1] Biagioni P. et al., Rep. Prog. Phys. 75, 024402 (2012).

[2] Toma A. et al., Nano Letters 15, 386-391 (2015).

[3] Malerba M. et al., Scientific Reports 5, 16436 (2015).

[4] Huth F. et al., Nano Letters 13, 1065-1072 (2013). 